\title{
Frequent tracheal suctioning is associated with extubation failure in patients with successful spontaneous breathing trial: a single-center retrospective cohort study
}

\author{
Junpei Haruna ${ }^{1 *} \mathbb{D}$, Hiroomi Tatsumi ${ }^{1}$, Satoshi Kazuma ${ }^{1}$, Aki Sasaki $^{2}$ and Yoshiki Masuda ${ }^{1}$
}

\begin{abstract}
Background: Extubation failure, i.e., reintubation in ventilated patients, is a well-known risk factor for mortality and prolonged stay in the intensive care unit (ICU). Although sputum volume is a risk factor, the frequency of tracheal suctioning has not been validated as a predictor of reintubation. We conducted this study to examine whether frequent tracheal suctioning is a risk factor for reintubation.

Patients and methods: We included adult patients who were intubated for $>72 \mathrm{~h}$ in the ICU and extubated after completion of spontaneous breathing trial (SBT). We compared the characteristics and weaning-related variables, including the frequency of tracheal suctioning between patients who required reintubation within $24 \mathrm{~h}$ after extubation and those who did not, and examined the factors responsible for reintubation.

Results: Of the 400 patients enrolled, reintubation was required in 51 (12.8\%). The most common cause of reintubation was difficulty in sputum excretion (66.7\%). There were significant differences in sex, proportion of patients with chronic kidney disease, pneumonia, ICU admission type, the length of mechanical ventilation, and ICU stay between patients requiring reintubation and those who did not. Multivariate analysis showed frequent tracheal suction (> once every $2 \mathrm{~h}$ ) and the length of mechanical ventilation were independent factors for predicting reintubation.
\end{abstract}

Conclusion: We should examine the frequency of tracheal suctioning $>$ once every $2 \mathrm{~h}$ in addition to the length of mechanical ventilation before deciding to extubate after completion of SBT in patients intubated for $>72 \mathrm{~h}$ in the ICU.

Keywords: Reintubation, tracheal suctioning, intensive care unit

\section{Background}

Extubation failure, i.e., reintubation, is a known risk factor for mortality and prolonged intensive care unit (ICU) stay in patients who receive mechanical ventilation in the ICU $[1,2]$. Incidence of reintubation has been reported to be approximately $10 \%$, and therefore, we should keep in mind the risk of reintubation and aim for safe extubation

\footnotetext{
*Correspondence: jp.haruna@hotmail.co.jp

1 Department of Intensive Care Medicine, School of Medicine, Sapporo Medical University, South-1, West-16, Chuo-ku, Sapporo, Hokkaido 060-8543, Japan

Full list of author information is available at the end of the article
}

[3, 4]. Epstein has demonstrated that patients with advanced age, anemia, hypoalbuminemia, and chronic respiratory failure, and those undergoing highly invasive surgery carry a risk for reintubation [5]. Moreover, clinical tools for evaluating successful extubation, such as the rapid shallow breathing index (RSBI) [6], are available for spontaneous breathing trials (SBTs). Therefore, extubation should be tried carefully after confirming favorable results of SBT in mechanically ventilated patients. However, reintubations sometimes occur despite following established protocols [7]. 
The main causes of reintubation are difficulty in sputum excretion and airway obstructions [5]. Sputum excretion is challenging owing to the amount of sputum available for extraction and the ability to discharge it $[8$, 9]. Although it is difficult for ICU healthcare workers to quantitatively evaluate the amount of airway secretion when performing trachea suctioning, the frequency of tracheal suction during mechanical ventilation may be associated with the amount of tracheal secretions [10]. We hypothesized that the frequency of tracheal suctioning is associated with the amount of tracheal secretions. This study, therefore, elucidates whether reintubation is associated with the frequency of tracheal suctioning before extubation.

\section{Methods}

We performed a single-center, retrospective, observational study in a university hospital. We collected data from patients admitted to the ICU between January 2011 and December 2017. The study design and protocol were approved by the Institutional Review Board (IRB) of Sapporo Medical University (IRB authorized number: 322266). Owing to the observational nature of this study, the information was released on an opt-out basis.

We included patients ( $\geq 18$ years) who had been intubated for $>72 \mathrm{~h}$ in the ICU of Sapporo Medical University Hospital and extubated after confirming successful SBT results defined as follows: respiratory rate $<30 / \mathrm{min}, \mathrm{SpO}_{2}>94 \%$, heart rate $<140 \mathrm{bpm}$, no arrhythmia, no excessive increase in blood pressure and no effort breathing, under continuous positive airway pressure $\leq 5 \mathrm{cmH}_{2} \mathrm{O}$ or pressure support $\leq 5 \mathrm{cmH}_{2} \mathrm{O}$ or T-piece for $\geq 30$ min with inspiratory oxygen concentration $\left(\mathrm{FIO}_{2}\right) \leq 0.4$. SBT was performed when the patient fulfilled the condition described in Table 1, and the ultimate decision to extubate was made by the intensive-care clinician based on SBT data, as well as the patient's hemodynamic stability, responsiveness, ability to follow commands, the strength of cough, and the ability to clear secretions. The exclusion criteria were patients after cardiovascular surgery or those with a tracheostomy. Extubation failure was defined as reintubation within $24 \mathrm{~h}$ after extubation.

Information obtained from electronic medical records included age, sex, underlying disease, Charlson Comorbidity Index (CCI), patient category at ICU admission (postoperative or medical), ICU length of stay (LOS), Acute Physiology and Chronic Health Evaluation (APACHE) II score at ICU admission, Sequential Organ Failure Assessment score at ICU admission, ventilation days, 28-day mortality, delirium, RSBI, and frequency of tracheal suctioning. The primary outcome was the association between reintubation within $24 \mathrm{~h}$ after extubation and tracheal suctioning frequency (more than once every $2 \mathrm{~h}$ for up to $12 \mathrm{~h}$ ) $[5,11]$ before extubation.

The definition of tracheal suctioning is as follows.

1) The patient's effortful breathing is increased (increased respiratory workload findings).

2) Secretions are visible in the tracheal tube.

3) Coarse crackles that suggest the presence of secretions from the trachea to the right and left main

Table 1 Criteria for initiating SBT

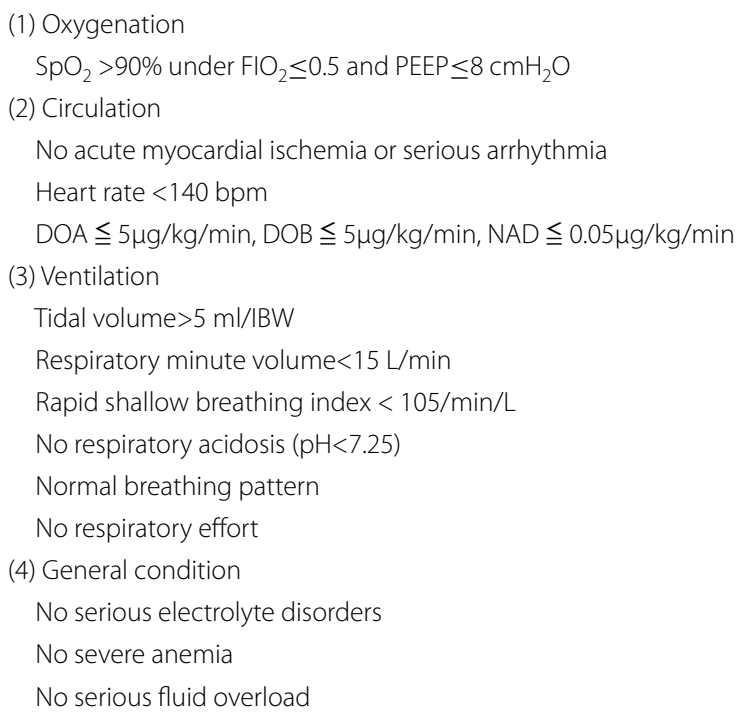


bronchi are heard on chest auscultation, or there is a decrease in breath sounds on chest auscultation.

4) Palpation of the chest wall reveals vibrations associated with the movement of gas.

5) Aspiration.

6) Blood gases and blood oxygen saturation rate $\left(\mathrm{SpO}_{2}\right)$ show hypoxemia.

7) An increase in airway pressure or a decrease in ventilation volumes.

Reintubation was performed when the patient had one or more of the following criteria: clinical signs of increased respiratory effort, upper airway obstruction, respiratory acidosis, hypoxemia $\left(\mathrm{SpO}_{2}<90 \%\right)$, decreased consciousness with unprotected upper airway $(\mathrm{GCS}<8)$, severe tachycardia, and continued tachypnea.

\section{Statistical analysis}

Categorical variables were expressed as numbers and percentages. Continuous variables were expressed as means and standard deviations. Chi-square tests were used for the nominal variables. The Mann-Whitney $U$ test was used for continuous variables. We hypothesized that the frequency of tracheal suctioning was an independent risk factor for reintubation. To evaluate this hypothesis, logistic regression analysis was performed to examine the odds ratio of the frequency of tracheal suctioning, adjusting for confounding factors that contribute to reintubation. We selected covariates based on the previous literature and clinical experience. Sex, CCI [7, 12], underlying pneumonia, APACHE II score at ICU admission [13], and ventilation days [12] were selected as confounding factors. The results of the multivariable analysis are shown with odds ratios (ORs), 95\% confidence intervals (CIs), and $p$-values. Statistical significance was set at $p<0.05$. Statistical analyses were performed using SPSS Statistics version 27 (IBM Corp., Armonk, NY, USA).

\section{Results}

During the study period, 601 patients received ventilator support for more than $72 \mathrm{~h}$. Fifty-one patients required reintubation within $24 \mathrm{~h}$ after extubation (reintubation group) (Fig. 1). The demographics of the patients and a comparison of vital signs and respiratory parameters in the reintubation and no reintubation groups are shown in Table 2.

Sex (male), admission type, ventilation days, and ICU LOS were significantly higher in the reintubation group. Furthermore, comorbidity of chronic kidney disease and underlying pneumonia was significantly higher in the reintubation group. The mean time to reintubation was $492 \mathrm{~min}$. The most common cause of reintubation was difficulty in sputum excretion, followed by hypoxemia and airway obstruction.

The results of the logistic regression analysis adjusted for the pre-defined covariates to examine the effect of the frequency of tracheal suctioning on reintubation are shown in Table 3.

A higher frequency of tracheal suctioning, defined once every $2 \mathrm{~h}$ up to $12 \mathrm{~h}$ before the extubation trial, was an independent factor associated with reintubation $(\mathrm{OR}=$ 10.65 , 95\% CI $=4.60-24.62, p<.001)$. Moreover, ventilation days were also an independent factor associated with reintubation (OR, 1.33; 95\% CI $=1.19-1.45, p<.001)$.

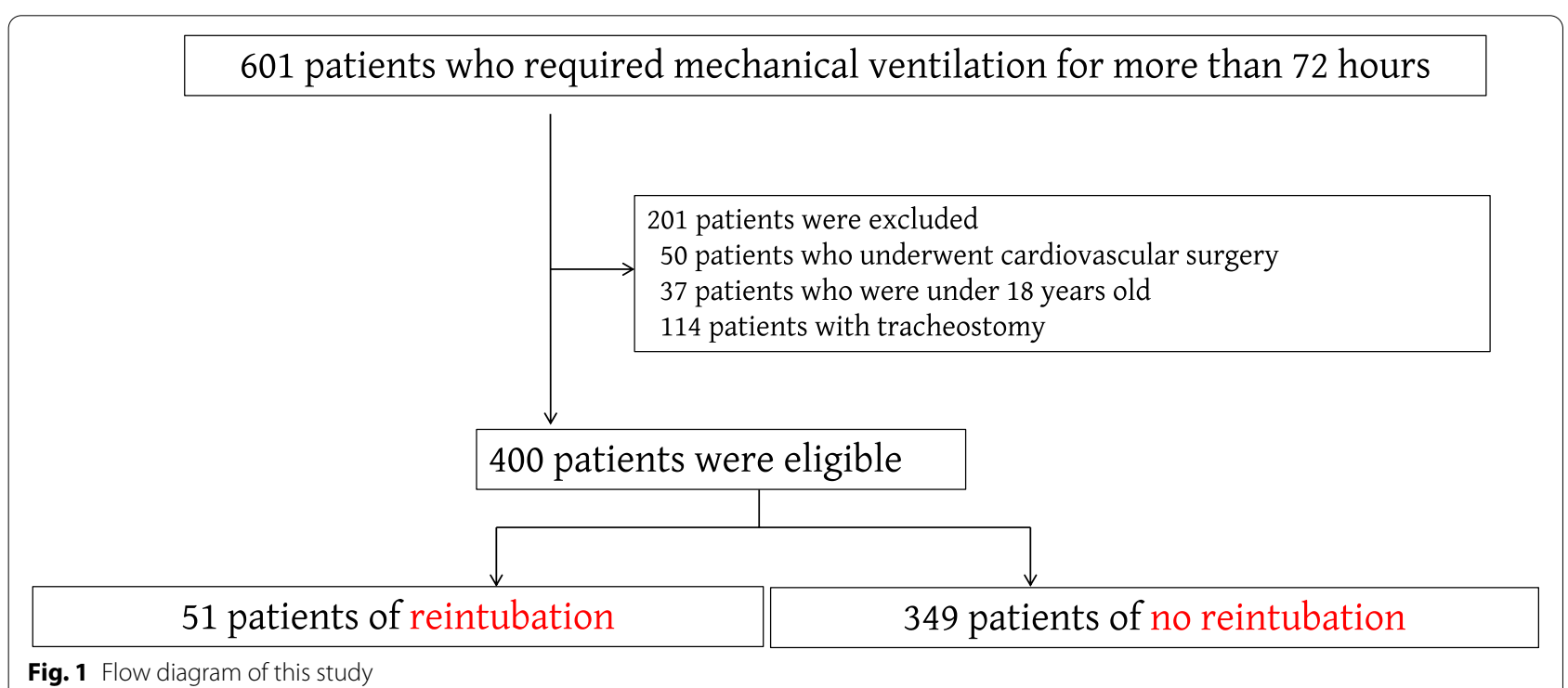

Fig. 1 Flow diagram of this study 
Table 2 Demographics of patients with and without reintubation

\begin{tabular}{|c|c|c|c|}
\hline & $\begin{array}{l}\text { Reintubation group } \\
n=51\end{array}$ & $\begin{array}{l}\text { No reintubation group } \\
n=349\end{array}$ & Pvalue \\
\hline Age (years), mean $\pm S D$ & $65.2 \pm 13.3$ & $64.3 \pm 14.2$ & 0.878 \\
\hline Gender, male, $n(\%)$ & $24(47.1)$ & $235(67.3)$ & 0.007 \\
\hline \multicolumn{4}{|l|}{ Comorbidities } \\
\hline Immune suppression & $4(7.8)$ & $26(2.9)$ & 1 \\
\hline Chronic liver disease & $6(11.8)$ & $27(7.7)$ & 0.4 \\
\hline Chronic kidney disease & $6(11.8)$ & $2(5.7)$ & $<.001$ \\
\hline Chronic heart failure & $5(9.8)$ & $60(17.2)$ & 0.225 \\
\hline Charlson Comorbidity Index & $1.6 \pm 1.8$ & $1.7 \pm 1.9$ & 0.73 \\
\hline \multicolumn{4}{|l|}{ Underlying diseases } \\
\hline Pneumonia & 17(33.3) & $47(13.5)$ & $<.001$ \\
\hline Sepsis & $7(13.7)$ & $36(10.3)$ & 0.468 \\
\hline ARDS & $4(7.8)$ & $16(4.6)$ & 0.303 \\
\hline heart failure & $3(5.9)$ & $12(3.4)$ & 0.421 \\
\hline Miscellaneous & 20(39.2) & 237(67.9) & $<.001$ \\
\hline \multicolumn{4}{|l|}{ Admission type } \\
\hline Surgical & 18(35.3) & $179(51.0)$ & 0.036 \\
\hline Medical & $33(64.7)$ & $170(49.0)$ & 0.036 \\
\hline SOFA at ICU admission, mean \pm SD & $6.4 \pm 2.5$ & $7.0 \pm 2.8$ & 0.048 \\
\hline APACHE II at ICU admission, mean \pm SD & $21.1 \pm 6.2$ & $20.8 \pm 5.8$ & 0.675 \\
\hline Delirium, $n(\%)$ & $14(27.5)$ & $105(30.1)$ & 0.746 \\
\hline RSBI & $39.6 \pm 12.9$ & $38.6 \pm 16.5$ & 0.322 \\
\hline Ventilation days (day) & $13.3 \pm 11.8$ & $5.0 \pm 2.7$ & $<.001$ \\
\hline Time to reintubation, mean $\pm S D$ & $492 \pm 470.4$ & - & \\
\hline ICU LOS (days), mean \pm SD & $14.2 \pm 12.5$ & $8.7 \pm 8.2$ & $<.001$ \\
\hline ICU mortality, n (\%) & 2(3.9) & $10(2.9)$ & 0.223 \\
\hline 28-day mortality, n (\%) & $6(11.8)$ & $17(4.9)$ & 0.097 \\
\hline \multicolumn{4}{|l|}{ Reason of reintubation, $n(\%)$} \\
\hline Difficulty in sputum excretion & $30(58.8)$ & - & \\
\hline Hypoxemia & $9(17.6)$ & - & \\
\hline Airway obstruction & $6(11.8)$ & - & \\
\hline Tachypnea & $4(7.8)$ & - & \\
\hline Consciousness disturbance & $1(2.0)$ & - & \\
\hline Hemosputum & $1(2.0)$ & - & \\
\hline
\end{tabular}

ICU intensive care unit, APACHE I/ Acute Physiology and Chronic Health Evaluation, SOFA Sequential Organ Failure Assessment, ARDS acute respiratory distress syndrome, LOS length of stay, RSBI rapid shallow breathing index

Table 3 Odds ratios regarding variables for extubation failure

\begin{tabular}{lllll}
\hline & $\begin{array}{l}\text { Regression } \\
\text { coefficient }\end{array}$ & OR & 95\%Cl & P value \\
\hline Male & 0.827 & 2.287 & $1.03-5.08$ & 0.042 \\
CCI & -0.133 & 0.875 & $0.70-1.09$ & 0.242 \\
Pneumonia & -0.585 & 0.557 & $0.225-1.381$ & 0.207 \\
APACHE II & -0.004 & 0.996 & $0.93-1.06$ & 0.899 \\
$\begin{array}{l}\text { Tracheal suction more } \\
\text { than once every 2 } \mathrm{h}\end{array}$ & 2.365 & 10.65 & $4.60-24.62$ & $<.001$ \\
Ventilation days & 0.272 & 1.31 & $1.19-1.44$ & $<.001$ \\
\hline
\end{tabular}

OR odds ratio, CCI Charlson Comorbidity Index, APACHE Il Acute Physiology and Chronic Health Evaluation

\section{Discussion}

In this study, we evaluated the association between reintubation and the frequency of tracheal suctioning in ventilated patients for more than $72 \mathrm{~h}$ and fulfilled extubation criteria such as SBT. The reintubation rate after extubation with successful SBT was $12.8 \%$, which was similar to a previous report [14]. The most common cause of reintubation was hypoxemia, followed by difficulty in sputum excretion. We found that tracheal suction more than once every $2 \mathrm{~h}$ was an independent factor associated with reintubation. The results of this study suggest that it may be useful to include 
the frequency of tracheal suctioning before extubation as one of the criteria for extubation in ventilator management.

In general, airway problems are a risk factor for reintubation [15]. One of the most common airway problems is the inability to clear airway secretions $[14,16]$. The clearance of airway secretions has two components: the intensity of cough and the volume of airway secretions. An association between cough intensity and reintubation has been reported in relation to a decrease in peak expiratory flow (PEF); however, the procedure for measuring PEF [15] is cumbersome and difficult to perform during daily clinical practice. Conversely, it has been reported that a high volume of airway secretion is associated with reintubation $[2,14,17,18]$. However, it is difficult for ICU healthcare workers to measure the amount of airway secretions when performing tracheal suctioning, thereby limiting the prediction of reintubation based on the amount of sputum secreted. Another way for ICU healthcare workers to determine the amount of airway secretions is the frequency of tracheal suctioning. It has been reported that intensivists use lower levels of airway secretion as an adjunctive indicator of successful extubation $[14,19]$. In this study, the frequency of tracheal suctioning was associated with reintubation. Therefore, it may be useful to decide extubation based on the frequency of tracheal suctioning. Respiratory physiotherapy may be useful to prevent reintubation in patients requiring frequent tracheal suctioning to prevent respiratory complications and for excretion training of airway secretions $[4,20]$.

Our results showed that the duration of mechanical ventilation was longer in the reintubation group than in the no reintubation group. Prolonged mechanical ventilation has been reported to negatively affect respiratory and limb (or extremities) muscle strength [21, 22] and is thought to result in ICU-acquired weakness. This study did not evaluate the onset of ICU-acquired weakness; however, it is important to provide multidisciplinary interventions such as early rehabilitation from the start of mechanical ventilation to achieve no reintubation. Particularly, when extubating patients with prolonged mechanical ventilation and a high volume of sputum, it should be considered that even with a successful SBT, extubation with respiratory physiotherapy is a strategy to reduce reintubation.

This study had several limitations. First, this is a retrospective, single-center, observational study. Therefore, our results may not be generalizable and need to be validated externally in future studies using a different dataset. Second, we used the nursing records obtained from an electronic patient information system, which may not reliably reflect actual clinical symptoms.

\section{Conclusion}

We found that a higher frequency of tracheal suctioning and ventilator days were associated with risk factors for reintubation in critically ill patients on mechanical ventilation. It is necessary to introduce protocols and other measures, such as clearer extubation criteria, to reduce reintubation in this population.

\begin{abstract}
Abbreviations
ARDS: Acute respiratory distress syndrome; AW: Acquired weakness; APACHE: Acute Physiology and Chronic Health Evaluation; CCl: Charlson Comorbidity Index; $\mathrm{Cl}$ : Confidence interval; $\mathrm{FlO}_{2}$ : Fractional inspired oxygen concentration; ICU: Intensive care unit; LOS: Length of stay; OR: Odds ratio; PEF: Peak expiratory flow; RSBI: Rapid shallow breathing index; SBT: Spontaneous breathing trial; SOFA: Sequential Organ Failure Assessment; $\mathrm{SpO}_{2}$ : Blood oxygen saturation rate.
\end{abstract}

\section{Acknowledgements}

We are grateful to Dr. Tomoko Sonoda for advice on statistical analysis.

\section{Authors' contributions}

$\mathrm{JH}, \mathrm{HT}, \mathrm{AS}$, and YM designed the work, collected and analyzed the data, and wrote the initial draft of the manuscript. SK contributed to the analysis and interpretation of the data and assisted in the preparation of the manuscript. All authors critically revised the manuscript and approved the final version for publication.

\section{Funding}

The authors have not received any funding

Availability of data and materials

The datasets analyzed during the current study are available from the corresponding author upon reasonable request.

\section{Declarations}

\section{Ethics approval and consent to participate}

The study design and protocol were approved by the Institutional Review Board (IRB) of Sapporo Medical University (IRB authorized number: 322-266). Owing to the observational nature of this study, the information was released on an opt-out basis.

\section{Consent for publication}

Not applicable.

\section{Competing interests}

YM reports grants from JIMRO and personal fees from MSD, Japan Blood Products Organization, and Asahi Kasei Pharma outside the submitted work. $H T$ received lecture fees from TSUMURA \& CO. The other authors declare that they have no competing interests.

\section{Author details}

${ }^{1}$ Department of Intensive Care Medicine, School of Medicine, Sapporo Medical University, South-1, West-16, Chuo-ku, Sapporo, Hokkaido 060-8543, Japan. ${ }^{2}$ Department of Nursing, Sapporo Medical University Hospital, Sapporo, Japan.

Received: 31 October 2021 Revised: 3 January 2022 Accepted: 4 January 2022

Published online: 13 January 2022

\section{References}

1. Epstein SK, Ciubotaru RL, Wong JB. Effect of failed extubation on the outcome of mechanical ventilation. Chest. 1997;112:186-92. https://doi. org/10.1378/chest.112.1.186. 
2. Jaber S, Quintard H, Cinotti R, Asehnoune K, Arnal JM, Guitton C, et al. Risk factors and outcomes for airway failure versus non-airway failure in the intensive care unit: a multicenter observational study of 1514 extubation procedures. Crit Care. 2018;22:236. https://doi.org/10.1186/ s13054-018-2150-6.

3. Thille AW, Richard JC, Brochard L. The decision to extubate in the intensive care unit. Am J Respir Crit Care Med. 2013;187:1294-302. https://doi. org/10.1164/rccm.201208-1523Cl.

4. Girard TD, Alhazzani W, Kress JP, Ouellette DR, Schmidt GA, Truwit JD, et al. An official American Thoracic Society/American College of Chest Physicians clinical practice guideline: liberation from mechanical ventilation in critically ill adults. Rehabilitation protocols, ventilator liberation protocols, and cuff leak tests. Am J Respir Crit Care Med. 2017;195:120-33. https:// doi.org/10.1164/rccm.201610-2075ST.

5. Epstein SK. Decision to extubate. Intensive Care Med. 2002;28:535-46. https://doi.org/10.1007/s00134-002-1268-8.

6. Yang KL, Tobin MJ. A prospective study of indexes predicting the outcome of trials of weaning from mechanical ventilation. N Engl J Med. 1991;324:1445-50. https://doi.org/10.1056/NEJM199105233242101.

7. Thille AW, Cortés-Puch I, Esteban A. Weaning from the ventilator and extubation in ICU. Curr Opin Crit Care. 2013;19:57-64. https://doi.org/10. 1097/MCC.0b013e32835c5095.

8. Cooper RM, O'Sullivan E, Popat M, Behringer E, Hagberg CA. Difficult Airway Society guidelines for the management of tracheal extubation. Anaesthesia. 2013;68:217. https://doi.org/10.1111/anae.12139.

9. Smailes ST, McVicar AJ, Martin R. Cough strength, secretions and extubation outcome in burn patients who have passed a spontaneous breathing trial. Burns. 2013;39:236-42. https://doi.org/10.1016/j.burns.2012.09. 028.

10. Matilde INE, Eid RAC, Nunes AF, Ambrozin ARP, Moura RH, Carnieli-Cazati $D$, et al. Bronchial hygiene techniques in patients on mechanical ventilation: what are used and why? Einstein (São Paulo). 2018;16:eAO3856. https://doi.org/10.1590/s1679-45082018ao3856.

11. Lombardi FS, Cotoia A, Petta R, Schultz M, Cinnella G, Horn J. Prediction of extubation failure in Intensive Care Unit: systematic review of parameters investigated. Minerva Anestesiol. 2019;85:298-307. https://doi.org/10. 23736/S0375-9393.18.12627-7.

12. Thille AW, Boissier F, Ben Ghezala H, Razazi K, Mekontso-Dessap A, BrunBuisson C. Risk factors for and prediction by caregivers of extubation failure in ICU patients: a prospective study. Crit Care Med. 2015;43:613-20. https://doi.org/10.1097/CCM.0000000000000748.

13. Ferrer M, Valencia M, Nicolas JM, Bernadich O, Badia JR, Torres A. Early noninvasive ventilation averts extubation failure in patients at risk: a randomized trial. Am J Respir Crit Care Med. 2006;173:164-70. https://doi. org/10.1164/rccm.200505-7180C.

14. Khamiees M, Raju P, DeGirolamo A, Amoateng-Adjepong Y, Manthous CA. Predictors of extubation outcome in patients who have ly completed a spontaneous breathing trial. Chest. 2001;120:1262-70. https://doi.org/10. 1378/chest.120.4.1262.

15. Smina M, Salam A, Khamiees M, Gada P, Amoateng-Adjepong $Y$, Manthous CA. Cough peak flows and extubation outcomes. Chest. 2003;124:262-8. https://doi.org/10.1378/chest.124.1.262.

16. Gonçalves MR, Honrado T, Winck JC, Paiva JA. Effects of mechanical insufflation-exsufflation in preventing respiratory failure after extubation: a randomized controlled trial. Crit Care. 2012;16:R48. https://doi.org/10. 1186/cc11249.

17. Salam A, Tilluckdharry L, Amoateng-Adjepong Y, Manthous CA. Neurologic status, cough, secretions and extubation outcomes. Intensive Care Med. 2004;30:1334-9. https://doi.org/10.1007/s00134-004-2231-7.

18. Mokhlesi B, Tulaimat A, Gluckman TJ, Wang Y, Evans AT, Corbridge TC. Predicting extubation failure after completion of a spontaneous breathing trial. Respir Care. 2007:52:1710-7.

19. Tulaimat A, Mokhlesi B. Accuracy and reliability of extubation decisions by intensivists. Respir Care. 2011;56:920-7. https://doi.org/10.4187/respc are.00851

20. Wang TH, Wu CP, Wang LY. Chest physiotherapy with early mobilization may improve extubation outcome in critically ill patients in the intensive care units. Clin Respir J. 2018;12:2613-21. https://doi.org/10.1111/crj. 12965.

21. De Jonghe B, Bastuji-Garin S, Durand MC, Malissin I, Rodrigues P, Cerf C, et al. Respiratory weakness is associated with limb weakness and delayed weaning in critical illness. Crit Care Med. 2007;35:2007-15. https://doi. org/10.1097/01.ccm.0000281450.01881.d8.

22. De Jonghe B, Sharshar T, Lefaucheur JP, Authier FJ, Durand-Zaleski I, Boussarsar $M$, et al. Paresis acquired in the intensive care unit: a prospective multicenter study. JAMA. 2002;288:2859-67. https://doi.org/10.1001/ jama.288.22.2859.

\section{Publisher's Note}

Springer Nature remains neutral with regard to jurisdictional claims in published maps and institutional affiliations.

\section{Submit your manuscript to a SpringerOpen ${ }^{\circ}$ journal and benefit from:}

- Convenient online submission

- Rigorous peer review

- Open access: articles freely available online

- High visibility within the field

- Retaining the copyright to your article

Submit your next manuscript at $\boldsymbol{\nabla}$ springeropen.com 